\title{
ON THE PRESERVATION OF BAIRE CATEGORY UNDER PREIMAGES
}

\author{
DOMINIKUS NOLL
}

(Communicated by Dennis Burke)

\begin{abstract}
We discuss the problem of preservation of Baire category under continuous and feebly open preimages. We obtain a solution by imposing a completeness condition on the fibres $f^{-1}(y)$ of the function $f$ under consideration. Based on a theorem on the invariance of residuality under continuous and nearly feebly open images, we also derive a result concerning the preservation of category under continuous and nearly feebly open preimages. We end up with an open mapping theorem for functions $f$ of this kind defined on a Čech complete space.
\end{abstract}

\section{INTRODUCTION}

In this paper we consider the problem of preservation of Baire category under continuous preimages. Let $E, F$ be topological spaces and let $f: E \rightarrow F$ be a continuous and feebly open surjection (i.e. int $f(V) \neq \varnothing$ for nonempty open $V$ in $E$ ). Suppose $F$ is a Baire space. Under what conditions is $E$ a Baire space? Clearly a positive answer requires some additional information since every space $E$ may be collapsed to a point. A natural idea is to impose category assumptions on the fibres $f^{-1}(y)$ of $f$. This has been done by Frolik (see $\left[\mathrm{F}_{2}\right]$, [HM]). He obtains a positive answer in the case where $E$ is assumed to have a countable pseudo-base and the fibres $f^{-1}(y)$ are second category spaces apart from a first category subset of $F$. Here we seek solutions to the problem which avoid the countability assumption on the space $E$. We obtain such a solution by imposing a somewhat stronger condition on the fibres $f^{-1}(y)$, which we call fibre-completeness, and which in particular includes the case where the fibres $f^{-1}(y)$ of $f$ are compact. We prove that Baire category is preserved under continuous, feebly open and fibre-complete preimages.

In the second part of our paper we discuss the related problem of preservation of Baire category under continuous and nearly feebly open preimages (i.e. int $\overline{f(V)} \neq \varnothing$ for nonempty open $V$ in $E$ ). We prove that if $f: E \rightarrow F$ is a continuous and nearly feebly open surjection from a Čech complete space $E$

Received by the editors April 25, 1988 and, in revised form, October 31, 1988.

1980 Mathematics Subject Classification (1985 Revision). Primary 54C10, 46A30, 02 G20.

Key words and phrases. Fibre-completeness, (nearly) feebly open, (nearly) feebly continuous, Čech completeness, open mapping theorem, preimages of Baire spaces. 
to a completely regular space $F$, then for every dense Baire subspace $G$ of $F$ the preimage $f^{-1}(G)$ is a dense Baire subspace of $E$.

\section{PRELIMINARIES}

Our terminology is based on the book [E]. In addition, the following notions need special mentioning.

A function $f: E \rightarrow F$ is called (nearly) feebly continuous if for every open subset $V$ of $F, f^{-1}(V) \neq \varnothing$ implies int $f^{-1}(V) \neq \varnothing$ (resp. int $\left.\overline{f^{-1}(V)} \neq \varnothing\right)$. Dually, $f$ is called (nearly) feebly open if for every nonempty open set $U$ in $E$ the set int $f(U)$ (resp. int $\overline{f(U)}$ ) is nonempty.

Let $E$ be a topological space. A pair $(\varphi, T)$ consisting of a tree $T$ of height $\aleph_{0}$ and a mapping $\varphi$ with domain $T$ is called a web on $E$ if the following conditions are satisfied:

(i) $\{\varphi(t): t \in T\}$ is a $\pi$-base for $E$;

(ii) for fixed $t \in T$ the set $\left\{\varphi(s): t<_{T} s \in T\right\}$ is a $\pi$-base for $\varphi(t)$.

Recall that a family $\mathfrak{B}$ of open subsets of a space $E$ is called a $\pi$-base for $E$ if every nonempty open $U$ in $E$ contains some nonempty $V \in \mathfrak{B}$.

\section{FibRE-COMPLETENESS}

Let $E, F$ be topological spaces and let $f: E \rightarrow F$ be a function. $f$ is called fibre-complete if there exists a web $(\varphi, T)$ on $E$ such that for every $y \in F$ and every cofinal branch $\left(t_{n}\right)$ in $T$ (i.e. $t_{n}<_{T} t_{n+1}$ for all $n$ ) having $\varphi\left(t_{n}\right) \cap f^{-1}(y) \neq \varnothing$ for every $n$, the intersection $\bigcap\left\{\varphi\left(t_{n}\right): n \in \mathbf{N}\right\}$ is as well nonempty.

Let us consider some examples illuminating our definition.

(1) Let $E$ be a regular space and suppose that $f: E \rightarrow F$ has countably compact or sequentially compact or pseudo-compact fibres. Then $f$ is fibrecomplete. This follows easily by taking as $T$ the tree consisting of all finite sequences $\left(U_{0}, \ldots, U_{n}\right)$ of open subsets of $E$ having $\bar{U}_{i} \subset U_{i-1}$ and by setting $\varphi\left(U_{0}, \ldots, U_{n}\right)=U_{n}$.

(2) Let $f: E \rightarrow F, g: F \rightarrow G$ be continuous fibre-complete functions. Suppose $f$ is feebly open. Then $g \circ f$ is again fibre-complete. Indeed, suppose the webs $(\varphi, T),(\psi, S)$ are given for $f, g$ respectively in accordance with the definition of fibre-completeness. Let $R$ be the tree of height $\aleph_{0}$ consisting of all finite sequences $\left(\left(t_{1}, s_{1}\right), \ldots,\left(t_{n}, s_{n}\right)\right)$, where $t_{1}<_{T} \cdots<_{T} t_{n}, s_{1}<_{S} \cdots<_{S} s_{n}$, and with $f\left(\varphi\left(t_{i}\right)\right) \subset \psi\left(s_{i}\right) \subset f\left(\varphi\left(t_{i-1}\right)\right)$. Let $R$ be ordered in the natural way, and let $\chi$ be defined by $\chi\left(\left(t_{1}, s_{1}\right), \ldots,\left(t_{n}, s_{n}\right)\right)=\varphi\left(t_{n}\right)$. Then $(\chi, R)$ is a web on $E$ which makes $g \circ f$ a fibre-complete function.

\section{THE FEEBLY OPEN CASE}

In this section we obtain the mentioned result concerning the preservation of Baire category under continuous feebly open preimages. 
Theorem 1. Let $E, F$ be topological spaces and let $f: E \rightarrow F$ be a feebly open and fibre-complete function such that

$$
\begin{aligned}
& \text { for every nonempty open set } U \text { in } E \text { and every nonempty open } \\
& \text { set } V \subset \operatorname{int} f(U) \text {, the set } U \cap \operatorname{int} f^{-1}(V) \text { is nonempty. }
\end{aligned}
$$

Suppose that $F$ is a Baire space. Then so is $E$.

Proof. Our proof uses the Banach-Mazur game characterization of Baire category. Recall that in the Banach-Mazur game players I and II alternatively choose nonempty open sets $V_{0}, V_{1}, V_{2}, \ldots$ with $V_{i+1} \subset V_{i}$. Player II is said to win the game if the $V_{i}$ have some point on common, otherwise player I is winning. Formally, the game may be described by means of strategies for the players, as for instance done in [T]. Here we use the variant of the game where both players have a complete knowledge of all the previous moves at every stage. With these preparations we may now recall that a space $E$ is Baire if and only if for every strategy $\Xi$ for player I there exists a strategy $\Theta$ for player II such that II playing with $\Theta$ wins against I playing with $\Xi$.

Let $(\varphi, T)$ be given on $E$ in accordance with the definition of fibrecompleteness. Let $\Xi$ be a strategy for player I on the space $E$. We have to find a strategy $\Theta$ for player II such that II playing with $\Theta$ wins against I playing with $\Xi$. We define a strategy $\Xi^{\prime}$ for player I on the space $F$ using induction.

First $\Xi^{\prime}(\varnothing)$ has to be defined. Let $U_{0}=\Xi(\varnothing)$; then $U_{0}$ is a nonempty open set in $E$. Using condition (i) of a web, we find some $t_{0} \in T$ having $\varnothing \neq \varphi\left(t_{0}\right) \subset U_{0}$. Now define $\Xi^{\prime}(\varnothing)=$ int $f\left(\varphi\left(t_{0}\right)\right)=V_{0}$.

Now let $V_{1}$ be a nonempty open subset of $V_{0}$. We have to define $\Xi^{\prime}\left(V_{0}, V_{1}\right)$. By condition $(*)$ the set $U_{1}=\varphi\left(t_{0}\right) \cap$ int $f^{-1}\left(V_{1}\right)$ is nonempty. Let $U_{2}=$ $\Xi\left(U_{0}, U_{1}\right)$; then $U_{2}$ is a nonempty open subset of $\varphi\left(t_{0}\right)$, hence there exists $t_{2} \in T$ with $t_{0}<_{T} t_{2}$ such that $\varnothing \neq \varphi\left(t_{2}\right) \subset U_{2}$. Now define $\Xi^{\prime}\left(V_{0}, V_{1}\right)=$ int $f\left(\varphi\left(t_{2}\right)\right)=V_{2}$.

Proceeding in this way we complete the definition of $\Xi^{\prime}$. Suppose this has been done. Since $F$ is a Baire space, there exists a strategy $\Theta^{\prime}$ for player II on $F$ such that II with $\Theta^{\prime}$ wins against I with $\Xi^{\prime}$. Let $V_{0}, V_{1}, V_{2}, \ldots$ represent the corresponding play, i.e. $V_{0}=\Xi^{\prime}(\varnothing), V_{1}=\Theta^{\prime}\left(V_{0}\right), V_{2}=\Xi^{\prime}\left(V_{0}, V_{1}\right), V_{3}=$ $\Theta^{\prime}\left(V_{0}, V_{1}, V_{2}\right)$, etc. By the construction of $\Xi^{\prime}$ there exist sequences $\left(t_{2 k}\right)$ and $\left(U_{k}\right)$ having

(1) $\varnothing \neq \varphi\left(t_{2 k}\right) \subset U_{2 k}, \quad t_{2 k}<_{T} t_{2 k+2}$;

(2) $U_{2 k+1}=\varphi\left(t_{2 k}\right) \cap \operatorname{int} f^{-1}\left(V_{2 k+1}\right) \neq \varnothing$;

(3) $U_{2 k}=\Xi\left(U_{0}, \ldots, U_{2 k-1}\right)$.

We define a strategy $\Theta$ for player II on $E$ by setting $\Theta\left(U_{0}, \ldots, U_{2 k}\right)=U_{2 k+1}$. Then $U_{0}, U_{1}, \ldots$ represents the game of II with $\Theta$ against I with $\Xi$. We claim that $\Theta$ is winning, from which the result follows.

Let $y \in \bigcap\left\{V_{n}: n \geq 0\right\}$. This implies $\varphi\left(t_{2 k}\right) \cap f^{-1}(y) \neq \varnothing$ for every $k$, so by the choice of $(\varphi, T)$ the intersection $\bigcap\left\{\varphi\left(t_{2 k}\right): k \geq 0\right\}$ is nonempty, and this 
clearly implies $\bigcap\left\{U_{n}: n \geq 0\right\} \neq \varnothing$, hence $\Theta$ does in fact win against $\Xi$. This ends our proof.

Corollary. Let $E, F$ be topological spaces and let $f: E \rightarrow F$ be a feebly open mapping such that either

(1) $f$ is injective and feebly continuous, or

(2) $f$ is fibre-complete, and for every open set $V$ in $F$ the set int $f^{-1}(V)$ is dense in $f^{-1}(V)$.

Suppose that $F$ is a Baire space. Then so is $E$.

Proof. It suffices to check condition $(*)$ in both cases. But this is easy to see in either case.

Remarks. (1) The first part of the statement of the corollary gives a positive solution to a problem posed by Neubrunn [Ne]. We mention that in [PS] this problem has also been solved under more restrictive conditions using a completely different technique.

(2) Condition $(*)$ as well as condition (2) in the corollary are both slightly stronger statements than mere feeble continuity. We do not know, however, whether feeble continuity alone is sufficient to obtain the conclusion of Theorem 1 .

\section{THE NEARLY FEEBLY OPEN CASE}

One may ask whether feebly openness of the function $f$ in Theorem 1 and its corollary may be replaced by near feebly openness. This is not the case even when $f$ is supposed to be a continuous and nearly open bijection. Indeed, consider the following example. Let $F$ be a normed Baire space and let $E$ be the set $F$ endowed with a finer normed topology such that $E$ is of the first category in itself. Then the identity mapping $i: E \rightarrow F$ is a continuous and nearly open (see [K, p. 24]) bijection such that $i^{-1}$ does not preserve category.

Although the results of $\S 2$ do not carry over to the nearly feebly open case, there is a positive result on the preservation of Baire category under continuous and nearly feebly open preimages which holds in a more special situation.

Theorem 2. Let $E$ be a Čech complete space and let $F$ be completely regular. Let $f: E \rightarrow F$ be a continuous and nearly feebly open surjection. Suppose that $G$ is a dense Baire subspace of $F$. Then $f^{-1}(G)$ is a dense Baire subspace of $E$.

The proof of this result is mainly based on the following Proposition, which is of interest in itself.

Proposition. Let $E$ be a Čech complete space and let $F$ be completely regular. Let $f: E \rightarrow F$ be a continuous dense and nearly feebly open function. Then $f$ maps residual subsets of $E$ onto residual subsets of $F$.

Proof. (1) Observe that it suffices to prove that $f(E)$ is a residual subset of $F$. For suppose this has been shown. Let $G$ be a dense $G_{\delta}$-set in $E$. Then $G$ is 
itself Čech complete and clearly $f \mid G: G \rightarrow F$ is continuous, dense and nearly feebly open. So the above weaker statement implies that $f(G)$ is residual in $F$. Hence we are led to prove that $f(E)$ is residual in $F$.

(2) Next observe that it suffices to consider the case where $f$ is a surjection and prove that $f(E)$ contains a dense Čech complete subspace $G$. Indeed, if this statement has been established, then we consider $f: E \rightarrow f(E)$, which is a continuous and nearly feebly open surjection. Our statement just mentioned implies that $f(E)$ contains a dense Čech complete subspace $G$. But $G$ is an absolute $G_{\delta}$, i.e. $G$ is a $G_{\delta}$-set in $F$ (see $\left[\mathrm{F}_{1}\right]$ ), and this gives the result. So we may assume that $f$ is a surjection and prove the existence of a dense Čech complete subspace $G$ of $F$.

(3) Observe that the open subsets $V$ of $E$ having $f(V) \subset$ int $\overline{f(V)}$ form a $\pi$-base for $E$. Indeed, if $U$ is a nonempty open set in $E$, then

$$
V=U \cap f^{-1}(\text { int } \overline{f(U)})
$$

is a nonempty open subset of $U$ with the mentioned property.

(4) Since $E$ is Čech complete, it admits a complete sequence $\left(\mathfrak{U}_{n}\right)$ of open coverings. Here 'complete' means that every filter $\mathfrak{F}$ on $E$ satisfying $\mathfrak{U}_{n} \cap \mathfrak{F} \neq \varnothing$ for all $n$ has a cluster point (see $\left.\left[\mathrm{F}_{1}\right]\right)$. Now let $\mathfrak{S}_{n}$ be the family of all open sets $V$ in $E$ which are contained in some element of $\mathfrak{U}_{n}$ and satisfy $f(V) \subset \operatorname{int} \overline{f(V)}$. Then $\mathfrak{S}_{n}$ is a $\pi$-base as a consequence of (3). Moreover, the sequence $\left(\mathfrak{S}_{n}\right)$ is again complete in the above sense.

Using induction over $n$ and transfinite induction at every stage, we may select subfamilies $\mathfrak{W}_{n}$ of the $\mathfrak{S}_{n}$, respectively, such that the following conditions are satisfied:

(1) for $W, W^{\prime} \in \mathfrak{W}_{n}, W \neq W^{\prime}$, we have int $\overline{f(W)} \cap \operatorname{int} \overline{f\left(W^{\prime}\right)}=\varnothing$;

(2) the sets $G_{n}=\bigcup\left\{\right.$ int $\left.\overline{f(W)}: W \in \mathfrak{W}_{n}\right\}$ are dense in $F$;

(3) for every $W \in \mathfrak{W}_{n+1}$ there exists $W^{\prime} \in \mathfrak{W}_{n}$ having $\bar{W} \subset W^{\prime}$.

Since $F$ is a Baire space, the set $G=\bigcap\left\{G_{n}: n \in \mathbf{N}\right\}$ is dense in $F$. We prove that $G$ is Čech complete. Let

$$
\mathfrak{D}_{n}=\left\{G \cap \operatorname{int} \overline{f(\bar{W})}: W \in \mathfrak{W}_{n}\right\} ;
$$

then $\left(\mathfrak{D}_{n}\right)$ is a nested sequence of relatively open coverings of $G$. It remains to check that $\left(\mathfrak{D}_{n}\right)$ is complete. So let $\mathfrak{F}$ be a filter on $G$ with $\mathfrak{F} \cap \mathfrak{D}_{n} \neq \varnothing$ for all $n$. We have to prove that $\mathfrak{F}$ has a cluster point in $G$.

Let $O_{n} \in \mathfrak{D}_{n}$ be elements of $\mathfrak{F}, O_{n}=G \cap$ int $\overline{f\left(W_{n}\right)}$. Clearly the sequence $\left(O_{n}\right)$ must be decreasing, i.e. $O_{n+1} \subset O_{n}$, and hence by (3) above, the same must be true for the sequence $\left(W_{n}\right)$. But note that each $W_{n}$ is contained in an element of $\mathfrak{U}_{n}$. Using this and condition (3), we conclude that the set $K=\bigcap\left\{W_{n}: n \in \mathbf{N}\right\}$ is compact and nonempty and possesses $\left(W_{n}\right)$ as a neighborhood base. Since $f\left(W_{n}\right) \subset$ int $\overline{f\left(W_{n}\right)}$, we deduce that the compact set $f(K)$ in $F$ has $\left(\operatorname{int}\left(f\left(W_{n}\right)\right)\right.$ as a neighborhood base in $F$. But notice that $\bigcap\left\{\right.$ int $\left.\overline{f\left(W_{n}\right)}: n \in \mathbf{N}\right\} \subset G$ by the construction of the $\mathfrak{W}_{n}$, so $f(K)$ is a subset 
of $G$ which has $\left(O_{n}\right)$ as a neighborhood base. Clearly this implies that $\mathfrak{F}$ has a cluster point in $f(K)$, for otherwise we could find, using the compactness of $f(K)$, an open neighborhood $U$ of $f(K)$ missing an element of $\mathfrak{F}$. Since some $O_{n}$ must be contained in $U$ and, on the other hand, all $O_{n}$ meet all the elements of $\mathfrak{F}$, this is impossible. This completes the proof.

Let us now give the proof of Theorem 2. Let $U$ be a nonempty open subset of $E$. We have to show that $f^{-1}(G) \cap U$ is of the second category in $U$. By observation (3) in the proof of the above proposition, we may assume that $f(U) \subset$ int $\overline{f(U)}$. Assume that $f^{-1}(G) \cap U$ is of the first category in $U$. Choose a residual subset $R$ of $U$ having $f^{-1}(G) \cap R=\varnothing$. But notice that $f \mid U: U \rightarrow \operatorname{int} \overline{f(U)}$ is a continuous dense and nearly feebly open mapping from a Čech complete space to a completely regular space. Therefore $f(R)$ is residual in int $\overline{f(U)}$. But $f(R) \cap G=\varnothing$, so $G \cap$ int $\overline{f(U)}$ must be a first category subset of int $\overline{f(U)}$, and this contradicts the fact that $G$ is a dense Baire subspace of $F$. This completes our argument.

\section{OPEN MAPPING THEOREM}

In this final section we establish a second application of our proposition from $\S 3$. We obtain a result on the generic openness of continuous nearly feebly open functions on Čech complete spaces. Recall that a function $f: E \rightarrow F$ is open at $x \in E$ if $f(V)$ is a neighborhood of $f(x)$ in $F$ whenever $V$ is a neighborhood of $x$ in $E$. Analogously, $f$ is called nearly open at $x \in E$ if for every neighborhood $V$ of $x$ in $E$, the set $\overline{f(V)}$ is a neighborhood of $f(x)$ in $F$.

Theorem 3. Let $E$ be a Čech complete space and let $F$ be a completely regular space. Let $f: E \rightarrow F$ be a continuous nearly feebly open bijection. Then $f$ is feebly open. Moreover, $f$ is open at every point $x$ of near openness.

Proof. Let $U$ be a nonempty open set in $E$ and let $V$ be a nonempty open subset of $U$ having $\bar{V} \subset U$ and $f(V) \subset$ int $\overline{f(V)}$. We prove that int $\overline{f(V)} \subset$ $f(U)$, from which the first part of our statement follows.

Let $z \in \operatorname{int} \overline{f(V)}, z=f(y)$. It suffices to prove $y \in \bar{V}$. So let $W$ be an open neighborhood of $y$. We have to prove $V \cap W \neq \varnothing$. By continuity we may assume that $f(W) \subset \operatorname{int} \overline{f(V)}$, so

$$
O=\operatorname{int} \overline{f(V)} \cap \operatorname{int} \overline{f(W)}
$$

is a nonempty open set in $F$. Let $V_{1}=V \cap f^{-1}(O), W_{1}=W \cap f^{-1}(O)$. By our proposition the sets $f\left(V_{1}\right)$ and $f\left(W_{1}\right)$ are both residual in $O$, hence they intersect. Indeed, $f \mid V_{1}: V_{1} \rightarrow O$ and $f \mid W_{1}: W_{1} \rightarrow O$ are continuous dense and nearly feebly open functions and hence the proposition applies. This ends the first part of our proof.

Now suppose that $x$ is a point of near openness of $f$. This means that $f(x) \in \operatorname{int} \overline{f(V)}$ for every neighborhood $V$ of $x$ in $E$. But now the statement 
follows immediately from the first part of the proof, since $x \in V \subset \bar{V} \subset U$ was shown to imply int $\overline{f(V)} \subset f(U)$.

Corollary 1. Let $E$ be a completely metrizable space and let $F$ be completely regular. Suppose $f: E \rightarrow F$ is a continuous and nearly feebly open bijection. Then $f$ is open at the points of a dense $G_{\delta}$-subset of $E$.

Proof. In view of the second part of the statement of Theorem 3 it suffices to show that $f$ is nearly open at the points of a dense $G_{\delta}$-subset of $E$. So let $\mathfrak{U}_{n}$ denote the family of all open subsets $U$ of $E$ having diameter $\leq 1 / n$ with respect to some fixed metric for $E$ and satisfying $f(U) \subset$ int $\overline{f(U)}$. Since every $\mathfrak{U}_{n}$ is a $\pi$-base for $E$, the sets $G_{n}=\bigcup \mathfrak{U}_{n}$ are open dense in $E$, so $G=\bigcap\left\{G_{n}: n \in \mathbf{N}\right\}$ is a dense $G_{\delta}$ in $E$. Clearly $f$ is nearly open at the points of $G$. This ends the proof.

In the following result, recall that a function $f: E \rightarrow F$ is called nearly continuous at $x \in E$ if for every neighborhood $U$ of $f(x)$ in $F, \overline{f^{-1}(U)}$ is a neighborhood of $x$ in $E$.

Corollary 2. Let $E, F$ be Čech complete spaces and let $f: E \rightarrow F$ be a function whose graph $G(f)$ is either closed or a $G_{\delta}$-set in $E \times F$. Suppose that for every open set $V$ in $F$ the set int $\overline{f^{-1}(V)}$ is dense in $f^{-1}(V)$, i.e.

$$
\overline{f^{-1}(V)}=\overline{\text { int } \overline{f^{-1}(V)}} \text {. }
$$

Then $f$ is feebly continuous; even more, for every open set $V$ in $F$, int $f^{-1}(V)$ is dense in $f^{-1}(V)$. Moreover, if $x \in E$ is a point of near continuity of $f$, then $f$ is in fact continuous at $x$.

Proof. Consider the mapping $g: G(f) \rightarrow E,(x, f(x)) \rightarrow x$. Clearly $g$ is a continuous bijection from a Čech complete space onto a completely regular space. We claim that $g$ is nearly feebly open.

Let $W$ be a nonempty open set in $G(f)$. Clearly $W$ may be chosen to be of the form

$$
W=G(f) \cap(U \times V),
$$

where $U$ is open in $E$ and $V$ is open in $F$. Observe that $U \cap f^{-1}(V) \neq$ $\varnothing$. Since, by assumption, int $\overline{f^{-1}(V)}$ is dense in $\overline{f^{-1}(V)}$, we deduce that $U \cap$ int $\overline{f^{-1}(V)}$ is nonempty. Now observe that $g(W)=U \cap f^{-1}(V)$, so $U \cap$ int $\overline{f^{-1}(V)}$ is contained in $\overline{g(W)}$, proving that $g$ is nearly feebly open.

Theorem 3 implies that $g$ is feebly open. We may say even more; for every nonempty open set $W$ in $G(f)$, the interior of $g(W)$ is a dense subset of $g(W)$. Clearly this gives us the above feeble continuity statement on $f$ claimed in the corollary. Moreover, if $x$ is a point of near continuity of $f$, then $(x, f(x))$ turns out to be a point of near openness of $g$, so the second part of the statement follows as well.

Remark. Theorem 3 is closely related with our open mapping theorem $\left[\mathrm{N}_{1}\right.$, Theorem 1], while its Corollary corresponds with [ $\mathrm{N}_{2}$, Theorem 2]. 


\section{REFERENCES}

[E] R. Engelking, General topology, Warszawa, 1976.

[ $\left.F_{1}\right]$ Z. Frolík, Generalizations of the $G_{\delta}$-property of complete metric spaces, Czech. Math. J. 10 (85) (1960), 359-378.

$\left[\mathrm{F}_{2}\right]$ _ Remarks concerning the invariance of Baire spaces under mappings, Czech. Math. J. 11 (86), (1961), 381-385.

[HM] R. C. Haworth and R. A. McCoy, Baire spaces, Diss. Math. CXLI.

[K] G. Köthe, Topological vector spaces II, Springer-Verlag, New York, 1979.

[Ne] T. Neubrunn, A note on mappings of Baire spaces, Math. Slovaca 27 (1977), 173-176. Erratum: Math. Slovaca 27 (1977), 442.

[ $\mathrm{N}_{1}$ ] D. Noll, Open mapping theorems in topological spaces, Czech. Math. J. 35 (110), (1985), 373-384.

[ $\mathrm{N}_{2}$ ] _ _ Baire spaces and graph theorems, Proc. Amer. Math. Soc. 96, (1986), 141-151.

[PS] Z. Piotrowski and A. Szymanski, Some remarks on category in topological spaces, Proc. Amer. Math. Soc. 101 (1987), 156-160.

[T] F. Topsoe, Topological games and Cech completeness. General Topology and its Relations to modern Analysis and Algebra V, Fifth Prague Top. Symp., 1981, pp. 613-630.

Universität Stuttgart, Mathematisches Institut B, Pfaffenwaldring 57, 7000 Stuttgart 80, West Germany 